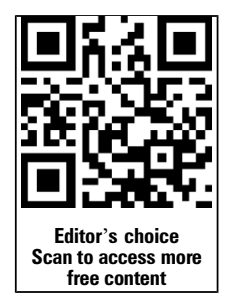

Scan to access mo free content

\title{
Ultrasound in the diagnosis of peripheral neuropathy: structure meets function in the neuromuscular clinic
}

\author{
Elena Gallardo, ${ }^{1,2}$ Yu-ichi Noto, ${ }^{3}$ Neil G Simon ${ }^{4,5}$
}

${ }^{1}$ Service of Radiology,

University Hospital Marqués de Valdecilla; Instituto de

Investigación Marqués de Valdecilla (IDIVAL), Santander, Spain

${ }^{2}$ University of Cantabria (UC); and Centro de Investigación

Biomédica en Red de

Enfermedades

Neurodegenerativas (CIBERNED), Santander, Spain ${ }^{3}$ Department of Neurology, Graduate School of Medical

Science, Kyoto Prefectural University of Medicine, Japan

${ }^{4}$ Prince of Wales Clinical School, University of New South Wales, Australia

${ }^{5}$ Central Clinical School, The University of Sydney, Australia

\section{Correspondence to}

Dr Neil G Simon, Prince of Wales Clinical School, University of New South Wales, Australia;

n.simon@unsw.edu.au

Received 16 October 2014 Revised 31 December 2014 Accepted 8 January 2015

Published Online First

4 February 2015

\section{ABSTRACT}

Peripheral nerve ultrasound (US) has emerged as a promising technique for the diagnosis of peripheral nerve disorders. While most experience with US has been reported in the context of nerve entrapment syndromes, the role of US in the diagnosis of peripheral neuropathy (PN) has recently been explored. Distinctive US findings have been reported in patients with hereditary, immunemediated, infectious and axonal PN; US may add complementary information to neurophysiological studies in the diagnostic work-up of PN. This review describes the characteristic US findings in PN reported to date and a classification of abnormal nerve US patterns in PN is proposed. Closer scrutiny of nerve abnormalities beyond assessment of nerve calibre may allow for more accurate diagnostic classification of PN, as well as contribute to the understanding of the intersection of structure and function in PN.

\section{INTRODUCTION}

Peripheral neuropathy (PN) contributes significantly to the neurological burden of disease worldwide. ${ }^{12}$ Prevalence of PN is increasing, particularly when associated with the growing population affected by diabetes, ${ }^{3}$ and the rising incidence of drug-induced neuropathy associated with chemotherapy and antiretroviral drugs. ${ }^{4} 5$

Traditionally, the diagnostic work-up of $\mathrm{PN}$ involves delineating a pattern of clinical involvement through history and examination, with the diagnosis confirmed by neurophysiological studies. In some situations, the clinical features and associated comorbidities may be enough to diagnose PN without further investigations, ${ }^{6}$ although grading of severity and monitoring of progression often includes neurophysiological assessment. However, the diagnosis of PN by clinical and neurophysiological grounds alone may be difficult, particularly in those patients with atypical or proximal demyelinating PN. ${ }^{7}$ It may also be difficult to distinguish acquired from inherited demyelinating PN. ${ }^{8}$ Hence, there is a need to develop novel strategies to aid in the diagnosis and monitoring of patients with PN, in particular the demyelinating forms.

The peripheral nervous system was unavailable to imaging modalities prior to the 1990s because of insufficient resolution and poor discrimination of nerves from surrounding soft tissues. However, recent technical developments have allowed imaging techniques, including MR neurography (MRN) and ultrasound (US), to play an important role in the diagnostic algorithm of peripheral nerve disorders. MRN currently provides an excellent depiction of three-dimensional nerve anatomy and pathology, and development of diffusion tensor imaging and tractography may provide further functional data. ${ }^{10}$ US provides superior spatial resolution that has enabled detailed visualisation of even the smallest peripheral nerves. Evolution of high-frequency broadband transducers (up to $22 \mathrm{MHz}$ ), advances in image postprocessing and sensitive Doppler technology, that allows assessment of nerve vascularity without contrast administration, have improved the ability of US to detect anatomic details and subtle structural abnormalities in peripheral nerves. In addition, the acquisition of US is a real-time dynamic process and allows the examiner to explore the entire course of the nerve in a single sweep. Finally, US has the general advantages of being a painless, non-invasive and inexpensive technique. As such, US may be considered to be an optimal tool to look for structural nerve pathology, and hence serve as a complementary technique to clinical and neurophysiological diagnosis of patients with PN. This review will discuss the US features of $\mathrm{PN}$, focusing on situations in which US studies may make a positive contribution to the diagnosis.

\section{US FEATURES OF NORMAL PERIPHERAL NERVE}

The US features of peripheral nerves correspond to macroscopic and microscopic anatomy. ${ }^{11}$ Peripheral nerves are visualised on US as tubular structures with a characteristic fascicular appearance (figures 1 and 2). On longitudinal images, linear hypoechoic fascicles are seen, separated by bands of hyperechoic perineurial connective tissue. On axial images, the peripheral nerves demonstrate a 'honeycomb' appearance, with ovoid hypoechoic fascicles embedded in a hyperechoic background. The dense epineurial connective tissue surrounding the nerve is highly reflective of sound waves, which results in a hyperechoic rim that may provide a means of demarcating the nerve from surrounding structures.

There are some situations where the US appearance of normal nerves differs from a typical fascicular pattern. Nerves are more hypoechoic and demonstrate fewer or no fascicles in very proximal nerves, such as the brachial plexus and cervical nerve roots, ${ }^{12}$ because of reduced volume of connective tissue and more tightly packed fascicles. ${ }^{13}$ Echogenicity and fascicle number may also be reduced where they cross osteofibrous tunnels, such as the ulnar nerve in the cubital tunnel. ${ }^{14}$ The size 


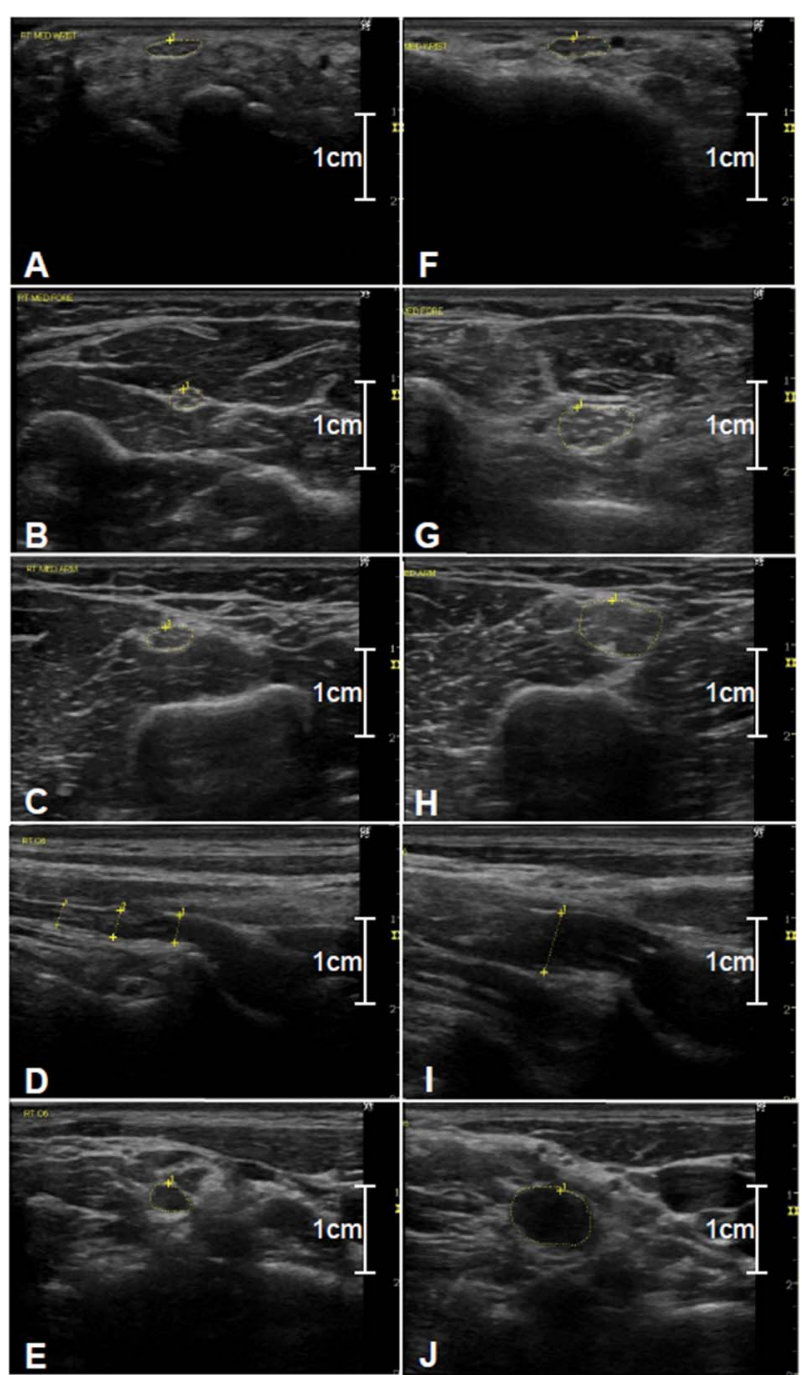

Figure 1 Ultrasound findings in Charcot-Marie-Tooth disease type $1 \mathrm{~A}$ (CMT1A). Ultrasound images of the median nerve (wrist-A mid-forearm-B, upper arm-C) and $C 6$ nerve root (longitudinal-D, axial-E) in a healthy subject and a patient with CMT1A (wrist-F, mid-forearm-G, upper arm- $\mathrm{H}, \mathrm{C} 6$ longitudinal-I, C6 axial-J) are depicted. Diffuse nerve enlargement was identified in the patient with CMT1A.

of peripheral nerves decreases slightly proximal to distal in the limb and it may be greater in entrapment sites in normal individuals. $^{14} 15$

Commercially available US units are able to assess most peripheral nerves of the upper limbs, lower limbs and brachial plexus. However, very deep nerves such as the proximal sciatic nerve may be difficult to image, and the lumbar and sacral plexus cannot be visualised using US.

\section{US FEATURES OF INJURED PERIPHERAL NERVE}

US findings following peripheral nerve injury converge on a number of common features. These changes include alterations in nerve size, nerve echotexture, definition of the epineurial margins, fascicle diameter and vascularity. Much of the literature describing peripheral nerve changes following nerve injury is based on assessment of entrapment neuropathies. ${ }^{16}$ In nerve compression, there may be focal nerve enlargement, loss of the internal fascicular appearance and decrease in nerve echogenicity. ${ }^{17}$

Nerve enlargement is most commonly quantified using crosssectional area (CSA) traced within the hyperechoic epineurial rim. CSA is a reliable measure with a good intraobserver and interobserver agreement and reproducibility; ${ }^{18}$ therefore, it has been most frequently used to quantify changes in neuropathy and reference values have been established for the major limb nerves in several anatomic locations and for the brachial plexus. ${ }^{15}{ }^{19-21}$ It is worthwhile noting that some studies have demonstrated that nerve size may be influenced by age, gender, body mass index and height. ${ }^{15} 19$ Temperature of the limb may also influence nerve calibre. ${ }^{22}$ As such, it is recommended that comparison groups in studies of nerve US are matched for those subject characteristics and standardised environmental conditions are employed during the study.

Measuring the size of individual nerve fascicles may also contribute important pathophysiological information for the nerve injury and $\mathrm{PN}$, although presently there is very little published data. ${ }^{23}$ Fascicle size can differ between individuals, nerves and anatomic regions of an individual nerve, and hence a standardised approach would be required to systematically study this.

Peripheral nerve echogenicity may be quantified by measuring the mean grey scale value of the nerve image. Alternatively, thresholding techniques may be applied to determine the proportion of the nerve that is relatively hypoechoic. ${ }^{142425}$ Data obtained using each of these approaches is specific to the US system being used and cannot be compared with data from another site unless the values are calibrated using a universal phantom.

Nerve vascularity, as measured by Doppler, may also provide insights into the pathophysiology of peripheral nerve disease. In normal nerves there is no detectable blood flow. ${ }^{26}$ Increased blood flow may be detected in compressive mononeuropathy and inflammatory $\mathrm{PN},{ }^{16}{ }^{27}$ possibly reflecting vascular proliferation precipitated by chronic trauma or inflammation (figure 3 ).

\section{US FINDINGS IN PN}

US is emerging as a valuable tool in the diagnosis of $\mathrm{PN}$ and it is in this field where it is anticipated that US will have a significant impact in rationalising the diagnostic pathway, potentially reducing the number of expensive investigations performed and focusing the use of expensive immunomodulatory therapies. ${ }^{28}$ In this section, the US findings documented to date in hereditary, immune-mediated, infectious and axonal neuropathies will be discussed.

\section{Hereditary neuropathies}

Charcot-Marie-Tooth disease

Charcot-Marie-Tooth disease (CMT) is a clinically and genetically heterogeneous hereditary neuropathy characterised by distal muscle atrophy, weakness and sensory loss with reduced tendon reflexes. More than 60 different causative gene mutations have been described. ${ }^{29}$ Nerve conduction studies still remain crucial both for the diagnosis and the classification of CMT (demyelinating type or axonal type), whereas US has emerged as a convenient technique to assess morphological changes of peripheral nerves in patients with CMT as a complement to the neurophysiological evaluation.

Nerve US findings of patients with CMT were first described in 1999 by Heinemeyer and Reimers ${ }^{30}$ They examined nerve diameter, but not CSA, in patients with CMT. They concluded that nerve diameter and echogenicity did not differ significantly between patients with CMT and healthy subjects, and noted that the visualisation of nerves with the $7.5 \mathrm{MHz}$ linear array probe was often difficult because of increased echogenicity of adjacent muscles in patients with CMT. The negative findings of this study may be explained by the limitations of resolution and 

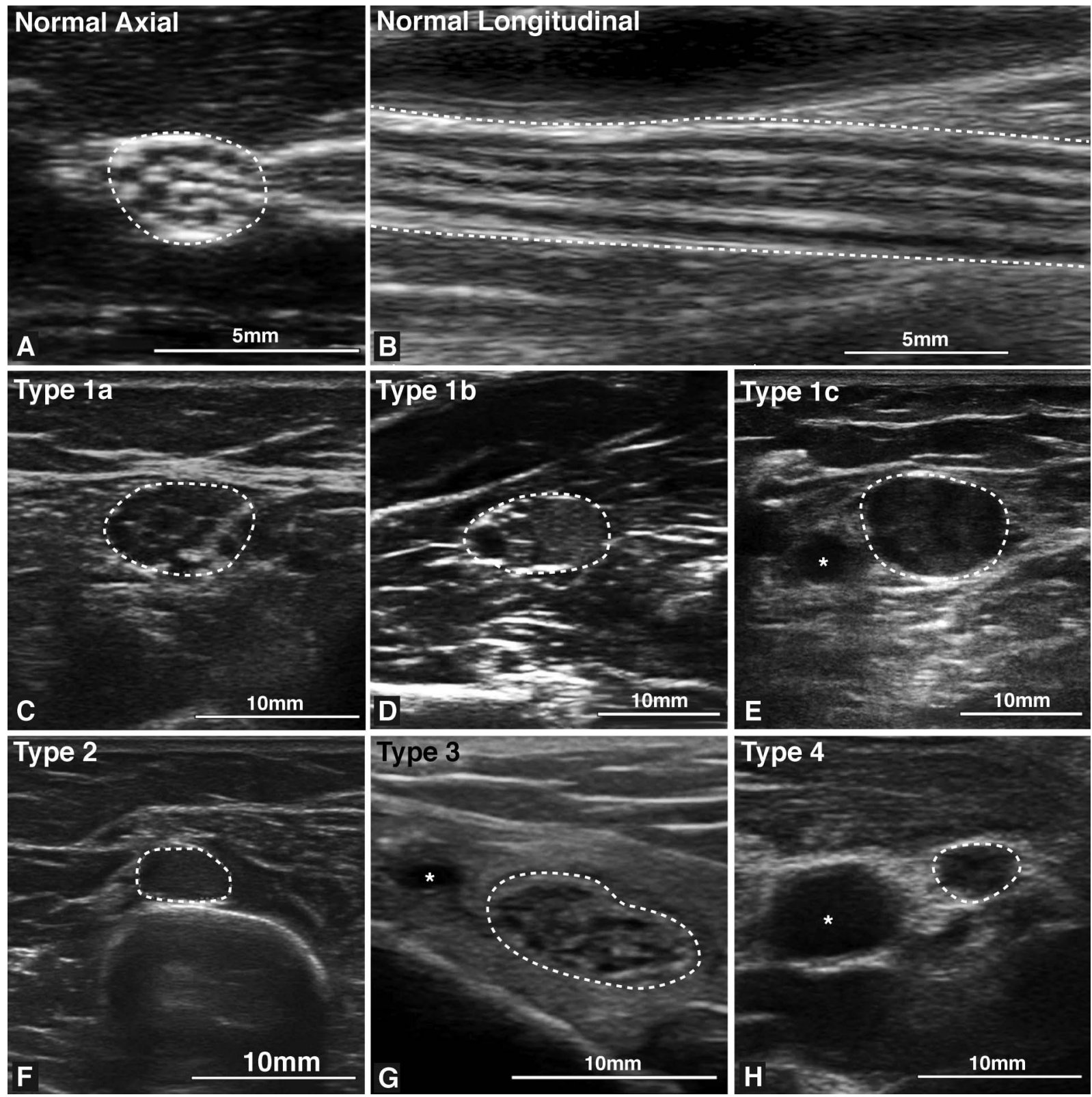

Figure 2 Patterns of nerve ultrasound changes in peripheral neuropathy (PN). Normal nerve ultrasound (US) appearances are shown in A (axial image of the median nerve in the forearm, cross-sectional area (CSA $7 \mathrm{~mm}^{2}$ ) and B (longitudinal image of the tibial nerve in the popliteal fossa, CSA $12 \mathrm{~mm}^{2}$ ) demonstrating a characteristic fascicular pattern. A number of patterns of nerve US abnormalities may be seen in PN and examples are shown in $\mathrm{C}-\mathrm{H}$. (C) An enlarged tibial nerve at the ankle (CSA $49 \mathrm{~mm}^{2}$ ) in a patient with CMT1A demonstrating heterogeneously enlarged hypoechoic fascicles (type 1a-uniform or heterogenous enlargement of hypoechoic fascicles). (D) An enlarged median nerve in the forearm (CSA $65 \mathrm{~mm}^{2}$ ) with mixed hyperechoic and hypoechoic fascicles in a patient with chronic inflammatory demyelinating polyradiculoneuropathy (CIDP) (type $1 \mathrm{~b}$ - mixed hyperechoic and hypoechoic fascicles). (E) An enlarged median nerve at the elbow (CSA $91 \mathrm{~mm}^{2}$ ) with disruption of the normal fascicular architecture in a patient with multifocal acquired demyelinating sensory and motor neuropathy (MADSAM) (type 1c - obliteration of normal fascicular architecture). (F) An enlarged radial nerve (CSA $27 \mathrm{~mm}^{2}$ ) in the spiral groove of a patient with CIDP, a region in which US may demonstrate a monofascicular or oligofascicular appearance of normal nerves (type 2-increase CSA in monofascicular nerve). (G) Enlargement of the tibial nerve at the ankle (CSA $95 \mathrm{~mm}^{2}$ ) in a patient with hypertrophic neuropathy, with prominent perineurial connective tissue and relatively normal fascicular calibre (type 3-increased CSA due to increased perineurial connective tissue). (H) The median nerve at the midpoint of the arm of a patient with amyloid neuropathy, demonstrating normal calibre (CSA $8 \mathrm{~mm}^{2}$ ) but with loss of normal fascicular architecture (type 4-normal CSA with altered echotexture).

recent studies using higher frequency probes $(\geq 12 \mathrm{MHz})$ have provided further information regarding nerve morphology in patients with various subtypes of CMT.

\section{CMT disease type 1A}

CMT1A, the most common form of demyelinating CMT, is caused by a duplication of PMP22 that encodes peripheral myelin protein 22 , a transmembrane protein in the compact myelin of the peripheral nerves. Schwann cells and abundant connective tissue around thinly myelinated axons ('onion bulbs') are the main features of pathology in CMT1A. In patients with CMT1A, nerve US reveals that the CSA of peripheral nerves, brachial plexus and nerve roots are larger than those in healthy subjects. Nerve CSA is uniformly increased throughout the course of the nerve, and the CSA and diameter of the C6 nerve root are also larger than those in controls (figure 1). Martinoli 

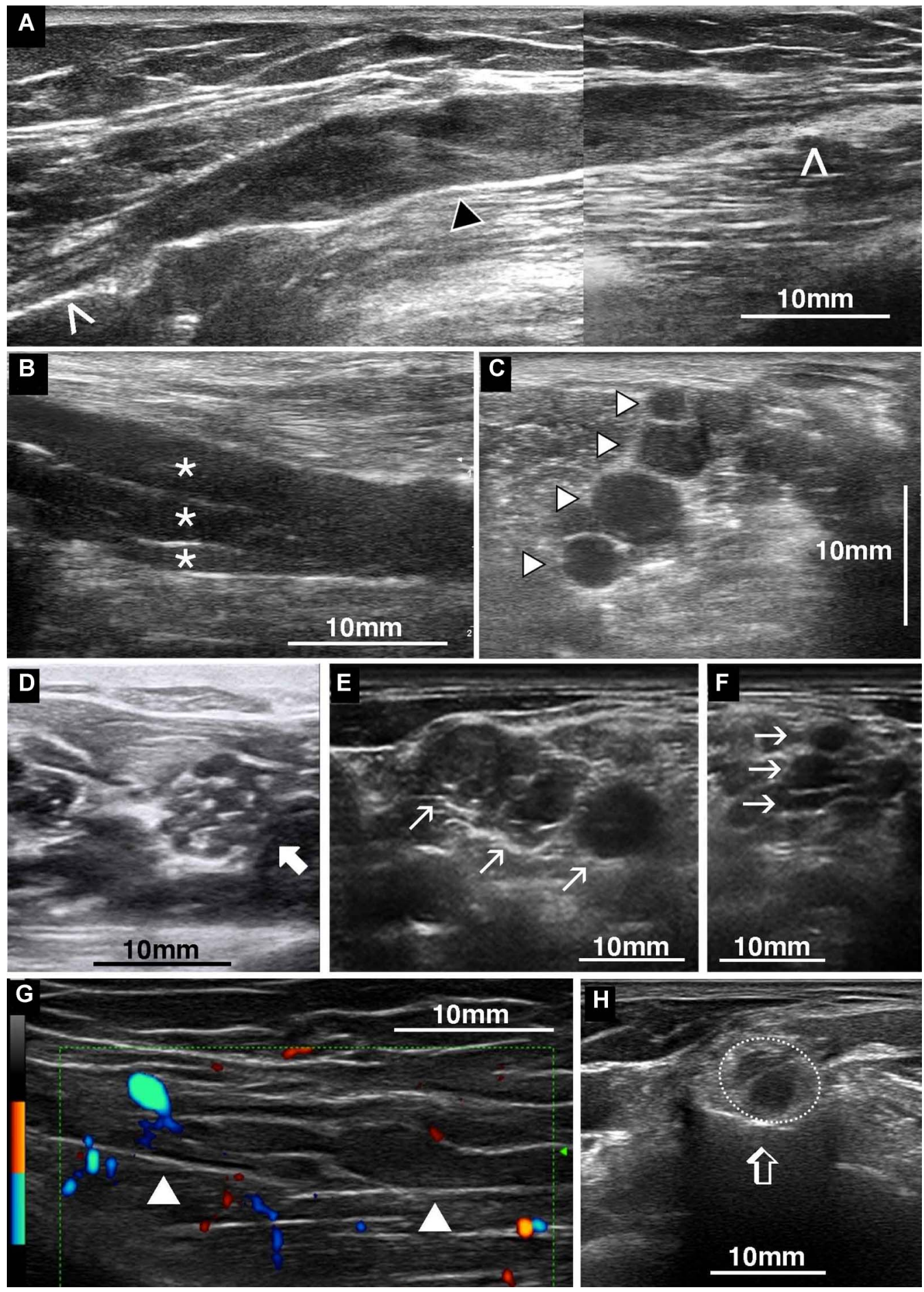

Figure 3 Ultrasound (US) abnormalities in chronic inflammatory demyelinating polyradiculoneuropathy (CIDP). A number of US abnormalities may be detected in patients with CIDP. (A) Focal enlargement and hypoechogenicity of the median nerve in the cubital fossa (black arrowhead) with a relatively normal fascicular pattern proximal and distal to the swelling $(\wedge)$.(B and C) Enlargement of the cervical nerve roots and brachial plexus (thin arrows), which is most commonly symmetric and may be associated with normal nerve calibre in more distal nerves. (D) Increased nerve cross-sectional area with prominent fascicular enlargement (thick arrow). In the patient, nerve enlargement was diffuse and involved all studied upper limb nerves. The cervical nerve roots (between anterior and middle scalene muscles) are depicted in a patient with multifocal acquired demyelinating sensory and motor neuropathy (MADSAM; E and F). US abnormalities were asymmetric with marked enlargement on the right (E) and relatively normal nerve calibre on the left (F). This corresponded with the clinical deficits, which were more severe in the right upper limb. (G) Increased nerve vascularity on Doppler US in the median nerve at the midpoint of the arm in a patient with CIDP. (H) The C6 nerve root in a patient with CIDP showing reduced definition of its epineurial margin.

et $a l^{31}$ reported that patients with CMT1A can be distinguished from those with other types of CMT (CMT2 and CMTX1) by either a larger CSA or a larger fascicular diameter in median nerves. Likewise, Noto et $a l^{32}$ demonstrated that CSA was also increased in the great auricular nerves and in C6 nerve roots in patients with CMT1A. Thus, nerve US findings demonstrate 
why CMT1A was previously classified as a hypertrophic neuropathy based just on nerve palpation.

Regarding the CSAs of the sural nerves, two conflicting descriptions have been reported. Pazzaglia $e a^{33}$ found that the CSA in the sural nerve was not increased in the majority of patients with CMT1A. Noto $e t a l^{32}$ found that the CSA in the sural nerve was increased in their population with CMT1A. One possible reason for this discrepancy can be attributed to the different methods of CSA measurement between their studies. Pazzaglia et al tracked the nerve circumference inside the hyperechoic rim, whereas Noto et al traced the nerve circumference including hyperechoic rim.

In terms of the correlation between US, clinical and electrophysiological findings in CMT1A, there is an inverse relationship between the CSA and the nerve conduction parameters, such as motor conduction velocity and compound muscle action potentials amplitude. ${ }^{23}{ }^{32}$ There is also a positive correlation between the CSAs in the median nerve and CMT neuropathy score (CMTNS) that quantifies the disease severity in patients with CMT1A. ${ }^{32}$ Taken together, in patients with CMT1A, the extent of nerve enlargement assessed by US paralleled not only the physiological function of peripheral nerves but also the clinical disease severity.

\section{CMT disease type $1 B$}

CMT1B, another demyelinating form of CMT, is caused by mutations of $M P Z$ gene that encodes myelin protein zero, a major constituent of peripheral myelin proteins. In a study involving a large family with CMT1B, enlargement of the median and vagus nerves was detected in affected family members. ${ }^{34}$ Conversely, sural nerve calibre was reduced, possibly reflecting length-dependent axonal loss.

\section{CMT disease type 2}

CMT2, a group of axonal forms of autosomal dominant CMT, includes more than 19 distinct types. Among them, the most common form is CMT2A that is caused by mutations of mitofusin 2 (MFN2) gene. In axonal neuropathies, axonal loss is predicted to result in decreased nerve caliber; however, median nerve CSA in patients with CMT2 is slightly larger than that of normal subjects. ${ }^{23} 31$ This discrepancy may be related to histopathological findings that include Schwann cell hyperplasia with pseudo onion bulb formations and endoneurial swelling seen in some genetic subtypes of CMT2. ${ }^{35}$

\section{CMT disease type $X 1$}

CMTX1, the second most common form of CMT, is caused by point mutations of gap junction-associated protein B1 (GJB1) gene, which encodes connexin-32 protein. Although a statistically significant difference was not demonstrated, the median nerve CSAs in patients with CMTX1 were larger than those in healthy subjects in one study, but were smaller in another report. ${ }^{23}{ }^{31}$ Both studies included a small number of patients; therefore, a further study with larger number of patients will be needed.

With the accumulation of future studies of US findings in various types of CMT, nerve US in combination with results of nerve conduction studies may provide tools to facilitate more targeted gene analysis in patients with suspected CMT. Nerve US is also useful for the diagnosis of hypertrophic-type CMT in the rare instance when compound muscle action potentials are not evoked in demyelinating CMT due to severe atrophy in distal muscles or marked increase in stimulation threshold.
Hereditary neuropathy with liability to pressure palsies

Hereditary Neuropathy with liability to pressure palsies (HNPP) is caused by a deletion of PMP22 and nerve biopsies in such patients reveal focal thickening of myelin, that is, tomacula. Beekman and Visser $^{36}$ first reported focal and multiple nerve enlargements in a patient with HNPP not only at typical nerve entrapment sites but also outside the entrapment sites. Non-uniform nerve enlargement patterns have been reported in some studies of patients with HNPP. $^{23} 3738$ Gianneschi et $a l^{37}$ revealed that no morphometric changes were seen in the distal nerve segments where entrapment is unlikely while the distal motor latencies were increased. Morphological abnormalities identified on US were not always correlated to the neurophysiological parameters in patients with HNPP, unlike those in patients with CMT1A.

\section{Immune-mediated neuropathies}

Chronic inflammatory demyelinating polyradiculoneuropathy (CIDP) Typical CIDP

The clinical features of typical chronic inflammatory demyelinating polyradiculoneuropathy (CIDP) are well recognised, including progressive symmetric weakness involving proximal more than the distal muscles, sensory impairment and reduced or absent deep tendon reflexes. Histopathologically, nerves in patients with CIDP demonstrate segmental demyelination and remyelination resulting in onion bulb formation and varying degrees of interstitial oedema and endoneurial inflammation. ${ }^{39}$ There are also a number of atypical or variant presentations, such as multifocal acquired demyelinating sensory and motor neuropathy (MADSAM), sensory-predominant CIDP, and distal forms such as distal acquired demyelinating sensory neuropathy (DADS). ${ }^{40-42}$

In the majority of patients with CIDP, abnormalities are detected on nerve US. However, just as there is clinical variability, there is a wide range of nerve US findings reported in CIDP (figure 3). Increased CSA of peripheral nerves and/or cervical nerve roots is most frequently reported. ${ }^{43-48}$ Hypertrophy of the vagus nerve has also been reported in $\operatorname{CIDP}^{49} 50$

While nerve enlargement is frequently identified, there may be marked variability in the nerve CSA both between-patients and within a patient. In some cases there may be massive enlargement (figure 3), but in other patients nerve calibre may be normal or mildly enlarged. Variability in nerve enlargement may be seen when different nerves of the same patient are compared and along the course of the same nerve, and identifying intranerve and internerve variability of CSA may be of diagnostic benefit. ${ }^{51}$

Three separate classes of US morphological findings have been described in CIDP depending on the CSA and echogenicity. $^{52}$ Class 1 nerves were enlarged with hypoechoic fascicles. Class 2 nerves were enlarged with mixed hypoechoic and hyperechoic fascicles. Class 3 nerves were of normal calibre but demonstrated abnormal hyperechoic fascicles, which were less easily distinguished from the perineurial connective tissue. The nerve US patterns correlated with disease duration (class 3 was associated with longer disease duration). As such, variations in US findings in CIDP may reflect different pathophysiological stages of the disease, although further histopathological correlation is needed. As CIDP is a chronic, segmental disorder often with a relapsing course, it is expected that different classes of nerve changes may coexist in some patients.

Nerve vascularity may also be increased in patients with CIDP as assessed with Doppler US studies (figure 3). ${ }^{53}$ Nerve blood flow strongly correlates with cerebrospinal fluid protein and the number of enlarged nerves, suggesting that nerve vascularity may reflect disease activity. 
The correlation between the US findings and neurophysiology features or functional disability remains controversial, ${ }^{43} \quad 52$ although correlation between the extent of nerve enlargement and the duration of the disease has been reported. ${ }^{15}{ }^{52}$ More detailed comparisons between US, clinical and neurophysiological findings are needed to clarify this point. Nerve US abnormalities may improve with treatment response. ${ }^{45}$

\section{CIDP variants}

The extent and nature of US abnormalities in CIDP variants are less well defined, although changes overlap with typical CIDP.

\section{Multifocal acquired demyelinating sensory and motor neuropathy}

Patients with MADSAM present with asymmetric motor and sensory deficits, often with patchy neurophysiological abnormalities in nerve conduction studies. US demonstrates multifocal nerve enlargements (figure 3 ) that may be identified at sites of current or previous electrophysiological conduction blocks. ${ }^{54}$

\section{Distal acquired demyelinating symmetric neuropathy}

The US features of DADS have not been systematically studied. Neuropathy associated with antimyelin-associated glycoprotein (MAG) antibodies is most commonly categorised with DADS and one study has evaluated US findings in this patient population. ${ }^{55}$ Patchy enlargement of nerves was identified most commonly at entrapment sites. Of interest, distal nerve enlargement was not prominent and this contrasts with the characteristic neurophysiological findings of prominent slowing of distal nerve conduction. ${ }^{56}$

\section{POEMS syndrome}

The demyelinating neuropathy associated with POEMS (polyneuropathy, organomegaly, endocrinopathy, M-protein, skin changes) syndrome may be confused with CIDP early in the course of the disease. Distinguishing clinical features include poor treatment response and the associated systemic features that contribute to the acronym. Increased serum vascular endothelial growth factor is a marker of the disease. US may also help distinguish POEMS syndrome neuropathy from CIDP. In POEMS syndrome, nerve enlargement may be seen at sites of nerve entrapment but is uncommon in other parts of the nerve ${ }^{57}$ which is distinct from the findings in CIDP.

\section{Multifocal motor neuropathy}

Multifocal motor neuropathy (MMN) is a rare neuropathy characterised by slowly progressive limb weakness, most commonly starting in the distal upper limb, with most patients responding to treatment with intravenous immunoglobulin. From a practical perspective, in some cases $\mathrm{MMN}$ can be difficult to distinguish from patients with progressive muscular atrophy. US studies identify focal nerve enlargement in the majority of patients with $\mathrm{MMN}$, including in limbs without neurophysiological dysfunction. ${ }^{45} 5859$ This is in contrast to the mild reduction of nerve CSA seen in MND. ${ }^{60}$ As such, US studies have been suggested as one method to select appropriate patients for treatment. ${ }^{28}$

\section{Guillain-Barré syndrome}

Presently, there are few studies reporting the US findings in patients with Guillain-Barré syndrome (GBS) and no studies to date comparing demyelinating with axonal GBS variants. Nerve enlargement has been reported in $47-83 \%$ of patients with early GBS, and may be present in peripheral nerves and/or cervical nerve roots. ${ }^{15} 61$ The distribution of nerve changes may be patchy within an individual ${ }^{61}$ and may be seen early before neurophysiological changes have developed. ${ }^{15}$ Alterations of the fascicular architecture have been reported, with heterogeneous focal enlargement of single fascicles noted in one case report. $^{62}$

In a detailed study, Gallardo et $a l^{61}$ described clinical, neurophysiological and US findings in six consecutive early GBS patients, with pathological correlation with autopsy material in two patients. US of the cervical nerve roots, and major upper and lower limb peripheral nerves was reported. US abnormalities were only detected in $8.8 \%$ of the scanned nerves, however, cervical nerve root abnormalities were identified in the majority of patients, consisting of increased CSA and reduced definition of epineurial margins. Indistinct margins of cervical nerve roots was a novel US finding and was correlated with nerve oedema demonstrated on corresponding pathological studies, which suggested that US findings may reflect the pathogenesis of the disease.

While longitudinal studies are generally lacking, a single case report demonstrated that US changes normalised during recovery, in keeping with clinical and neurophysiological improvement. $^{62}$ However, increased nerve CSA was identified in patients with residual deficits years after the onset of GBS, but these US changes did not correlate with functional disability. ${ }^{63}$

\section{Infectious polyneuropathies}

Leprosy is the most common infectious cause of neuropathy worldwide. Nerve enlargement and loss of fascicular pattern are seen on US. ${ }^{27}{ }^{64}$ These abnormalities are most frequent at common sites of nerve entrapment, in particular the cubital tunnel. However, generally the nerve enlargement tends to be more extensive and less circumscribed. Thickened and hypoechoic epineurium is a characteristic finding. Immunologically mediated reversal reactions are a common cause of skin and nerve injury in leprosy, and Doppler US at this stage may demonstrate increased nerve vascularity, which suggests rapid progression of nerve damage and a poor prognosis. ${ }^{64}$

\section{Axonal neuropathy}

The role of US may be less well defined in axonal PN. Intuitively, one may expect reduced CSA in axonal PN due to loss of myelinated fibres. However, this is seldom apparent with the exception of modest reduction of nerve calibre in amyotrophic lateral sclerosis (ALS). ${ }^{6065}{ }^{66}$ In fact, US of axonal PN may detect nerve enlargement in approximately $20 \%$ of patients. ${ }^{15}$

Studies of US in diabetic PN, most of them focused on the evaluation of tibial and median nerves, have demonstrated evidence of nerve and fascicle enlargement, and loss of fascicular pattern. ${ }^{67-70}$ Correlation with electrophysiological parameters has been noted in some but not in all studies. Specifically, inverse relationships between nerve CSA and compound muscle action potential amplitude and motor nerve conduction velocity have been identified. ${ }^{69}$ Increased water content due to conversion of glucose into sorbitol in the nerve was suggested as a cause of the increased nerve CSA. ${ }^{67}$ Nerve enlargement may be an interesting marker of diabetic PN severity in future studies, although it is noted that this finding has not been reported in all studies of diabetic PN. ${ }^{15} 71$

Oxaliplatin-induced neuropathy, which has axonal features on neurophysiological studies, ${ }^{72}$ is not associated with reduced CSA on US but rather nerve enlargement at sites of nerve entrapment-a finding suggesting increased susceptibility to mechanical nerve injury. ${ }^{73}$ 


\section{Nerve tumours}

When only imaging data is considered, hypertrophic neuropathy may be mistaken for a peripheral nerve tumour and vice versa, particularly when nerve enlargement is segmental or when tumours are multifocal, for example, neurofibromatosis, multiple schwannomatosis and intraneural perineurioma. ${ }^{74} 75$ A comprehensive review of US in peripheral nerve tumours is beyond the scope of this review (and readers are directed to specific reviews on the topic ${ }^{76} 77$ ). However, characteristic lesion features are noted in some peripheral nerve tumours, which may help distinguish them from hypertrophic neuropathy. A comprehensive US study of proximal and distal peripheral nerves combined with clinical and neurophysiological information should satisfactorily distinguish each disease process; however, fascicular nerve biopsy may sometimes be needed.

\section{PRACTICAL APPROACH TO US DIAGNOSIS Mapping nerve abnormalities}

An important aspect of the US examination of suspected PN is analysis of the topographic distribution of nerve abnormalities. This includes: the number of nerves involved (diffuse, multifocal or localised); the presence of a proximal or distal predominance; and the uniformity of the involvement along the course of the nerve. Comprehensive US assessment of the peripheral nervous system is the recommended approach, which may include examination of the brachial plexus, upper extremity nerves (median, radial and ulnar) and lower extremity nerves (femoral, sciatic, peroneal, tibial and sural), with the composition of the study guided by the clinical phenotype but also including clinically unaffected regions.

\section{Distinguishing CMT from CIDP}

One issue experienced in the neuromuscular clinic is distinguishing some acquired neuropathies from hereditary demyelinating neuropathies and broad diagnostic test batteries and empirical treatment trials are often employed to confirm a diagnosis. Nerve US may contribute to the diagnosis of demyelinating neuropathies in a number of ways.

US may help differentiate CMT from mimicking acquired demyelinating neuropathies, such as in those patients in whom clinical features and nerve conduction studies remain inconclusive. Evaluation of CSAs at intermediate nerve segments helped distinguish demyelinating CMT from CIDP because the CSAs in patients with demyelinating CMT were uniformly enlarged while those in patients with CIDP demonstrated variable enlargement. ${ }^{78}$ However, care must be taken because patients with demyelinating CMT other than CMT1A do not always exhibit nerve enlargement. $^{32}$

In addition, US studies facilitate assessment of proximal nerve segments that may be difficult to assess with nerve conduction studies, and hence may improve the detection of PN with demyelinating features in a predominantly proximal distribution.

\section{Identifying the contribution of nerve compression}

Despite non-specific findings in patients with axonal PN, US does have an important role in the diagnosis or exclusion of superimposed entrapment neuropathy in these patients, which can be difficult to diagnose using electrophysiological studies alone. US is able to confirm the diagnosis of compressive neuropathy and to rule out anatomical contributions to nerve injury.

As an example, increased CSA of the median nerve without change in wrist-to-forearm ratio might be compatible with diabetic PN, while it would not be indicative of carpal tunnel syndrome. ${ }^{79}$ Many causes of PN do not lead to altered nerve morphology on US, but may predispose to secondary entrapment neuropathy. For example, $70 \%$ of patients with systemic sclerosis and sensory complaints have US evidence of carpal tunnel syndrome or ulnar neuropathy at the elbow. ${ }^{80}$

\section{FUTURE DIRECTIONS: BEYOND CSA}

Recent publications have demonstrated the utility of US in the work-up of patients with PN. However, many studies to date have examined heterogeneous populations. In addition, most studies have focused on the measurement of CSA at different sites, and there is overlap of values with healthy populations. Unlike findings in entrapment mononeuropathy, there is frequently no correlation between the changes in nerve calibre in $\mathrm{PN}$ and clinical severity, although detailed clinical information is often not reported. More detailed exploration of the US changes in PN may provide a more powerful assessment of nerve pathology, which may help in the diagnosis of PN.

The high resolution of US allows accurate assessment of different morphological characteristics of the nerve independent of CSA and these features have seldom been mentioned in published studies. The features that may warrant further exploration include: fascicle diameter, fascicle-to-connective tissue ratio, epineurial demarcation and nerve blood flow. ${ }^{23} 5253$

\section{Proposed classification of nerve US abnormalities}

Although the angle of insonation and other technical factors impact on the nature of the nerve image acquired by US, information regarding the histopathological processes occurring within the nerve may be available with closer scrutiny of the US appearance of the nerve. Taking into account the different morphological aspects that can be evaluated at present, the previous reports and our personal observations, we propose the following patterns of nerve involvement (figure 2):

- Type 1: Increased CSA in multifascicular nerves due to fascicular enlargement.

- Type 1a: With uniform or heterogeneous enlargement of hypoechoic fascicles as seen in hereditary demyelinating neuropathies and CIDP.

- Type 1b: With mixed hyperechoic and hypoechoic fascicles as seen in longstanding CIDP.

- Type 1c: With obliteration of normal sonographic fascicular appearance as seen in inflammatory PN, nerve trauma, HNPP, leprosy and some axonal neuropathies; mild examples of this pattern are also common in entrapment sites in nerves of asymptomatic normal individuals.

- Type 2: Increased CSA in monofascicular nerves. This pattern may be seen in the brachial plexus and cervical nerve roots in inflammatory neuropathies, such as GBS and CIDP, and hereditary neuropathies such as CMT.

- Type 3: Increased CSA in multifascicular nerves due to increased perineurial connective tissue. This may be seen in unusual neuropathies, such as hypertrophic mononeuropathy and leprosy, and may contribute to US changes in diabetic neuropathy.

- Type 4: Normal CSA with fascicular enlargement or altered echotexture as seen in CIDP and deposition disorders such as amyloid neuropathy.

- Type 5: Decreased CSA. Reduced CSA has been reported in ALS and rarely in studies of patients with axonal PN.

\section{CONCLUSIONS}

US has a complementary role in the diagnosis of PN. US has the advantage of excellent resolution of superficial nerves, 
and the dynamic nature of image acquisition makes it a natural fit for the neuromuscular and electrodiagnostic clinics. Neurophysiological studies have long been considered to be an extension of the clinical examination. ${ }^{81}$ It is expected that a similar assertion will be increasingly relevant for US, and the addition of anatomic and structural information may provide important complementary diagnostic information.

Further, US may be useful to distinguish between different types of neuropathy, in particular the demyelinating neuropathies, by identifying patterns of morphological changes. Incorporating US features into diagnostic algorithms may rationalise the process of diagnostic testing with inherent time and cost savings. Exploration of nerve US features in addition to CSA may provide additional pathophysiological and diagnostic insights. Further correlation between US and MRI in PN may allow the roles of each of these techniques to be better delineated.

Presently, correlations between nerve morphology and electrophysiological function are emerging in demyelinating neuropathies, such as CMT and CIDP, and diabetic neuropathy; however, further exploration is needed to determine the relationship between structure and function in other neuropathy subtypes. Understanding of this emerging diagnostic tool will be better developed by detailed comparisons of US findings with clinical, neurophysiological and histopathological features, and this is recommended as a focus of future research.

Acknowledgements NGS gratefully acknowledges funding from the National Health and Medical Research Council and the Motor Neurone Disease Research Institute of Australia (grant \#1039520).

\section{Competing interests None.}

Provenance and peer review Commissioned; externally peer reviewed.

\section{REFERENCES}

1 Martyn CN, Hughes RA. Epidemiology of peripheral neuropathy. I Neurol Neurosurg Psychiatry 1997;62:310-18.

2 MacDonald BK, Cockerell OC, Sander JW, et al. The incidence and lifetime prevalence of neurological disorders in a prospective community-based study in the UK. Brain 2000;123:665-76.

3 Simmons Z, Feldman EL. Update on diabetic neuropathy. Curr Opin Neurol 2002;15:595-603.

4 Park SB, Goldstein D, Krishnan AV, et al. Chemotherapy-induced peripheral neurotoxicity: a critical analysis. CA Cancer I Clin 2013;63:419-37.

5 Ellis RJ, Rosario D, Clifford DB, et al. Continued high prevalence and adverse clinical impact of human immunodeficiency virus-associated sensory neuropathy in the era of combination antiretroviral therapy: the CHARTER study. Arch Neurol 2010:67:552-8.

6 Hughes RA. Diagnosis of chronic peripheral neuropathy. J Neurol Neurosurg Psychiatry 2001;71:147-8.

7 Latov N. Diagnosis and treatment of chronic acquired demyelinating polyneuropathies. Nat Rev Neurol 2014;10:435-46.

8 Neligan A, Reilly MM, Lunn MP. CIDP: mimics and chameleons. Pract Neurol 2014;14:399-408.

9 Eppenberger P, Andreisek G, Chhabra A. Magnetic resonance neurography: diffusion tensor imaging and future directions. NNeuroimag Clin N Am 2014; $24: 245-56$

10 Simon NG, Narvid J, Cage T, et al. Visualizing axon regeneration after peripheral nerve injury with magnetic resonance tractography. Neurology 2014;83:1382-4.

11 Fornage BD. Peripheral nerves of the extremities: imaging with US. Radiology 1988;167:179-82.

12 Simon NG, Cage T, Narvid J, et al. High-resolution ultrasonography and diffusion tensor tractography map normal nerve fascicles in relation to Schwannoma tissue prior to resection. J Neurosurg 2014;120:1113-7.

13 Sheppard DG, Iyer RB, Fenstermacher MJ. Brachial plexus: demonstration at US. Radiology 1998;208:402-6.

14 Simon NG, Ralph JW, Poncelet AN, et al. A comparison of ultrasonographic and electrophysiologic 'inching' in ulnar neuropathy at the elbow. Clin Neurophysiol 2014. doi:0.1016/j.clinph.2014.05.023

15 Zaidman CM, Al-Lozi M, Pestronk A. Peripheral nerve size in normals and patients with polyneuropathy: an ultrasound study. Muscle Nerve 2009;40:960-6.
16 Martinoli C, Bianchi S, Gandolfo N, et al. US of nerve entrapments in osteofibrous tunnels of the upper and lower limbs. Radiographics 2000;20:S199-213.

17 Cartwright MS, Walker FO. Neuromuscular ultrasound in common entrapment neuropathies. Muscle Nerve 2013:48:696-704.

18 Tagliafico A, Cadoni A, Fisci E, et al. Reliability of side-to-side ultrasound cross-sectional area measurements of lower extremity nerves in healthy subjects. Muscle Nerve 2012;46:717-22.

19 Cartwright MS, Passmore LV, Yoon JS, et al. Cross-sectional area reference values for nerve ultrasonography. Muscle Nerve 2008;37:566-71.

20 Haun DW, Cho JC, Kettner NW. Normative cross-sectional area of the C5-C8 nerve roots using ultrasonography. Ultrasound Med Biol 2010;36:1422-30.

21 Won SJ, Kim BJ, Park KS, et al. Measurement of cross-sectional area of cervical roots and brachial plexus trunks. Muscle Nerve 2012;46:711-16.

22 Ulasli AM, Tok F, Karaman A, et al. Nerve enlargement after cold exposure: a pilot study with ultrasound imaging. Muscle Nerve 2014;49:502-5.

23 Schreiber S, Oldag A, Kornblum C, et al. Sonography of the median nerve in CMT1A, CMT2A, CMTX, and HNPP. Muscle Nerve 2013;47:385-95.

24 Tagliafico A, Tagliafico G, Martinoli C. Nerve density: a new parameter to evaluate peripheral nerve pathology on ultrasound. Preliminary study. Ultrasound Med Biol 2010;36:1588-93.

25 Boom J, Visser LH. Quantitative assessment of nerve echogenicity: comparison of methods for evaluating nerve echogenicity in ulnar neuropathy at the elbow. Clin Neurophysiol 2012;123:1446-53.

26 Joy V, Therimadasamy AK, Chan YC, et al. Combined Doppler and B-mode sonography in carpal tunnel syndrome. J Neurol Sci 2011;308:16-20.

27 Jain S, Visser LH, Praveen TL, et al. High-resolution sonography: a new technique to detect nerve damage in leprosy. PLoS Negl Trop Dis 2009;3:e498.

28 Simon NG, Ayer G, Lomen-Hoerth C. Is IVlg therapy warranted in progressive lower motor neuron syndromes without conduction block? Neurology 2013;81:2116-20.

29 Rossor AM, Polke JM, Houlden $\mathrm{H}$, et al. Clinical implications of genetic advances in Charcot-Marie-Tooth disease. Nat Rev Neurol 2013:9:562-71.

30 Heinemeyer 0 , Reimers $C D$. Ultrasound of radial, ulnar, median, and sciatic nerves in healthy subjects and patients with hereditary motor and sensory neuropathies. Ultrasound Med Biol 1999;25:481-5.

31 Martinoli C, Schenone A, Bianchi S, et al. Sonography of the median nerve in Charcot-Marie-Tooth disease. Am J Roentgenol 2002;178:1553-6.

32 Noto YI, Shiga K, Tsuji Y, et al. Nerve ultrasound depicts peripheral nerve enlargement in patients with genetically distinct Charcot-Marie-Tooth disease. J Neurol Neurosurg Psychiatry 2014;86:378-84.

33 Pazzaglia C, Minciotti I, Coraci D, et al. Ultrasound assessment of sural nerve in Charcot-Marie-Tooth 1A neuropathy. Clin Neurophysiol 2013;124:1695-9.

34 Cartwright MS, Brown ME, Eulitt $\mathrm{P}$, et al. Diagnostic nerve ultrasound in Charcot-Marie-Tooth disease type 1B. Muscle Nerve 2009;40: 98-102.

35 Vallat JM, Ouvrier RA, Pollard JD, et al. Histopathological findings in hereditary motor and sensory neuropathy of axonal type with onset in early childhood associated with mitofusin 2 mutations. J Neuropath Exp Neurol 2008;67:1097-102.

36 Beekman R, Visser LH. Sonographic detection of diffuse peripheral nerve enlargement in hereditary neuropathy with liability to pressure palsies. I Clin Ultrasound 2002;30:433-6.

37 Ginanneschi F, Filippou G, Giannini F, et al. Sonographic and electrodiagnostic features of hereditary neuropathy with liability to pressure palsies. I Periph Nervous System 2012;17:391-8.

38 Hooper DR, Lawson W, Smith L, et al. Sonographic features in hereditary neuropathy with liability to pressure palsies. Muscle Nerve 2011;44: 862-7.

39 Dyck PJ, Lais AC, Ohta M, et al. Chronic inflammatory polyradiculoneuropathy. Mayo Clin Proc 1975;50:621-37.

40 Katz JS, Saperstein DS, Gronseth G, et al. Distal acquired demyelinating symmetric neuropathy. Neurology 2000;54:615-20.

41 Lewis RA, Sumner AJ, Brown MJ, et al. Multifocal demyelinating neuropathy with persistent conduction block. Neurology 1982;32:958-64.

42 Oh SJ, Joy JL, Kuruoglu R. "Chronic sensory demyelinating neuropathy": chronic inflammatory demyelinating polyneuropathy presenting as a pure sensory neuropathy. J Neurol Neurosurg Psychiatry 1992;55:677-80.

43 Kerasnoudis A, Pitarokoili K, Behrendt V, et al. Correlation of nerve ultrasound, electrophysiological and clinical findings in chronic inflammatory demyelinating polyneuropathy. J Neuroimaging 2014.

44 Kerasnoudis A, Pitarokoili K, Behrendt V, et al. Nerve ultrasound score in distinguishing chronic from acute inflammatory demyelinating polyneuropathy. Clin Neurophysiol 2014;125:635-41.

45 Zaidman CM, Harms MB, Pestronk A. Ultrasound of inherited vs. acquired demyelinating polyneuropathies. J Neurol 2013;260:3115-21.

46 Zaidman CM, Pestronk A. Nerve size in CIDP varies with disease activity and therapy response over time: a retrospective ultrasound study. Muscle Nerve 2014;50:733-8 
47 Goedee HS, Brekelmans GJF, van Asseldonk JTH, et al. High resolution sonography in the evaluation of the peripheral nervous system in polyneuropathy - a review of the literature. Eur J Neurol 2013;20:1342-51.

48 Matsuoka N, Kohriyama T, Ochi K, et al. Detection of cervical nerve root hypertrophy by ultrasonography in chronic inflammatory demyelinating polyradiculoneuropathy. J Neuro/ Sci 2004;219:15-21.

49 Grimm A, Thomaser AL, Peters N, et al. Vagal hypertrophy in immune-mediated neuropathy visualised with high-resolution ultrasound (HR-US). J Neurol Neurosurg Psychiatry 2014. doi:10.1136/jnnp-2014-308271

50 Jang JH, Cho CS, Yang KS, et al. Pattern analysis of nerve enlargement using ultrasonography in chronic inflammatory demyelinating polyneuropathy. Clin Neurophysiol 2014;125:1893-9.

51 Padua L, Martinoli C, Pazzaglia C, et al. Intra- and internerve cross-sectional area variability: new ultrasound measures. Muscle Nerve 2012;45:730-3.

52 Padua L, Granata G, Sabatelli $M$, et al. Heterogeneity of root and nerve ultrasound pattern in CIDP patients. Clin Neurophysiol 2014;125:160-5.

53 Goedee HS, Brekelmans GJ, Visser LH. Multifocal enlargement and increased vascularization of peripheral nerves detected by sonography in CIDP: a pilot study. Clin Neurophysiol 2014;125:154-9.

54 Scheidl E, Bohm J, Simo M, et al. Ultrasonography of MADSAM neuropathy: focal nerve enlargements at sites of existing and resolved conduction blocks. Neuromuscular Disord 2012;22:627-31.

55 Lucchetta M, Padua L, Granata G, et al. Nerve ultrasound findings in neuropathy associated with anti-myelin-associated glycoprotein antibodies. Eur J Neurol 2015;22:193-202.

56 Capasso M, Torrieri F, Di Muzio A, et al. Can electrophysiology differentiate polyneuropathy with anti-MAG/SGPG antibodies from chronic inflammatory demyelinating polyneuropathy? Clin Neurophysiol 2002;113:346-53.

57 Lucchetta M, Pazzaglia C, Granata G, et al. Ultrasound evaluation of peripheral neuropathy in POEMS syndrome. Muscle Nerve 2011;44:868-72.

58 Beekman $\mathrm{R}$, van den Berg LH, Franssen $\mathrm{H}$, et al. Ultrasonography shows extensive Inerve enlargements in multifocal motor neuropathy. Neurology 2005;65:305-7.

59 Kerasnoudis A, Pitarokoili K, Behrendt V, et al. Multifocal motor neuropathy: correlation of nerve ultrasound, electrophysiological and clinical findings. J Periph Nervous System 2014;19:165-74.

60 Cartwright MS, Walker FO, Griffin LP, et al. Peripheral nerve and muscle ultrasound in amyotrophic lateral sclerosis. Muscle Nerve 2011;44:346-51.

61 Gallardo E, Sedano MJ, Orizaola P, et al. Spinal nerve involvement in early Guillain-Barre syndrome: a clinico-electrophysiological, ultrasonographic and pathological study. Clin Neurophysio/ 2014. doi:10.1016/j.clinph.2014.06.051

62 Almeida V, Mariotti $P$, Veltri $S$, et al. Nerve ultrasound follow-up in a child with Guillain-Barre syndrome. Muscle Nerve 2012;46:270-5.

63 Kerasnoudis A, Pitarokoili K, Behrendt V, et al. Correlation of nerve ultrasound, electrophysiological, and clinical findings in post Guillain-Barre syndrome. J Periph Nervous System 2013;18:232-40.
64 Martinoli C, Derchi LE, Bertolotto M, et al. US and MR imaging of peripheral nerves in leprosy. Skel Radiol 2000;29:142-50.

65 Nodera H, Takamatsu N, Shimatani Y, et al. Thinning of cervical nerve roots and peripheral nerves in ALS as measured by sonography. Clin Neurophysiol 2014;125:1906-11.

66 Schreiber S, Abdulla S, Debska-Vielhaber G, et al. Peripheral nerve ultrasound in ALS phenotypes. Muscle Nerve 2014. doi:10.1002/mus.24431

67 Riazi S, Bril V, Perkins BA, et al. Can ultrasound of the tibial nerve detect diabetic peripheral neuropathy? A cross-sectional study. Diabetes Care 2012;35:2575-9.

68 Liu F, Zhu J, Wei M, et al. Preliminary evaluation of the sural nerve using $22-\mathrm{MHz}$ ultrasound: a new approach for evaluation of diabetic cutaneous neuropathy. PLOS ONE 2012;7:e32730.

69 Watanabe $T$, Ito $H$, Sekine A, et al. Sonographic evaluation of the peripheral nerve in diabetic patients: the relationship between nerve conduction studies, echo intensity, and cross-sectional area. J Ultrasound Med 2010;29:697-708.

70 Zheng Y, Wang L, Krupka TM, et al. The feasibility of using high frequency ultrasound to assess nerve ending neuropathy in patients with diabetic foot. Eur J Radiol 2013;82:512-7.

71 Hobson-Webb LD, Massey JM, Juel VC. Nerve ultrasound in diabetic polyneuropathy: correlation with clinical characteristics and electrodiagnostic testing. Muscle Nerve 2013;47:379-84.

72 Park SB, Lin CS, Krishnan AV, et al. Oxaliplatin-induced neurotoxicity: changes in axonal excitability precede development of neuropathy. Brain 2009;132:2712-23.

73 Briani C, Campagnolo M, Lucchetta M, et al. Ultrasound assessment of oxaliplatin-induced neuropathy and correlations with neurophysiologic findings. Eur J Neurol 2013;20:188-92.

74 Wang LM, Zhong YF, Zheng DF, et al. Intraneural perineurioma affecting multiple nerves: a case report and literature review. Int J Clin Exp Pathol 2014;7:3347-54.

75 Koontz NA, Wiens AL, Agarwal A, et al. Schwannomatosis: the overlooked neurofibromatosis? Am J Roentgenol 2013;200:W646-53.

76 Gruber H, Glodny B, Bendix N, et al. High-resolution ultrasound of peripheral neurogenic tumors. Eur Radiol 2007;17:2880-8.

77 Reynolds DL Jr, Jacobson JA, Inampudi P, et al. Sonographic characteristics of peripheral nerve sheath tumors. Am J Roentgenol 2004;182:741-4.

78 Sugimoto $\mathrm{T}$, Ochi $\mathrm{K}$, Hosomi $\mathrm{N}$, et al. Ultrasonographic nerve enlargement of the median and ulnar nerves and the cervical nerve roots in patients with demyelinating Charcot-Marie-Tooth disease: distinction from patients with chronic inflammatory demyelinating polyneuropathy. J Neurol 2013;260:2580-7.

79 Moon HI, Kwon HK, Kim L, et al. Ultrasonography of palm to elbow segment of median nerve in different degrees of diabetic polyneuropathy. Clin Neurophysiol 2014;125:844-8.

80 Tagliafico A, Panico N, Resmini E, et al. The role of ultrasound imaging in the evaluation of peripheral nerve in systemic sclerosis (scleroderma). Eur J Radiol 2011;77:377-82.

81 Simon NG. Dynamic muscle ultrasound-another extension of the clinical examination. Clin Neurophysiol 2014. doi:10.1016/j.clinph.2014.10.153 